\title{
Actividad física subaeróbica de bajo impacto: una estrategia para disminuir el deterioro del sistema muscular y mejorar la calidad de vida en personas de la tercera edad, en los (cpr) distrito de Pachacamac.
}

\section{Low-impact subaerobic physical activity: a strategy to decrease the deterioration of the muscular system and improve the quality of life in the elderly, in the (cpr) district of Pachacamac.}

\author{
Ronald Alarcón', Cecilia Abensur 2
}

\section{RESUMEN}

El envejecimiento y deterioro en la población de la tercera edad es natural con el paso del tiempo, y el desarrollo de la práctica frecuente de actividad física y deportiva se considera como algo muy saludable, capaz de prevenir ciertas enfermedades, tanto físicas como psicológicas, e incluso, tratarlas y rehabilitarlas, lo cual puede ser buena en las personas ancianas.

Si éste fuera el caso, ¿qué tipo de ejercicio físico deberían realizar las personas mayores y con qué frecuencia? El presente estudio propuso, mejorar la calidad de vida de la población de la tercera edad de los CPR de Pachacamac, mediante un innovador Programa Integral Físico, Psicologíco, Nutricional, Psicomotor, Psicosocial y Cognitivo el cual nos permitira evaluar, desarrollar actividades acuaticas aerobicas de bajo impacto de esta manera disminuir el deterioro muscular mejorando la calidad de vida.

Palabras claves: Actividad física, vida saludable, actividades acuáticas, programas integrales y calidad de vida

\section{ABSTRACT}

Aging and deterioration in the elderly population is natural over time, and the development of frequent practice of physical and sports activity is considered as something very healthy, capable of preventing certain diseases, both physical and psychological, and even treating and rehabilitating them, which can be good in the elderly.

If this were the case, what type of physical exercise should older people do and how often? The present study proposed to improve the quality of life of the elderly population of the CPR of Pachacamac, through an innovative Comprehensive Physical, Psychological, Nutritional, Psychomotor, Psychosocial and Cognitive Program which will allow us to evaluate, develop aerobic aquatic activities of low impact in this way decrease muscle deterioration improving quality of life.

Key words: Physical activity, healthy life, aquatic activities, comprehensive programs and quality of life 


\section{INTRODUCCIÓN}

El Distrito de Pachacamac cuenta con una Ordenanza Municipal 169-2016-MDP/C proyecto de Ordenanza que aprueba la Creación del Centro Integral del Adulto Mayor -CIAM al nuevo Marco Legal Vigente Ley № 30490, sin embargo se puede visualizar en el portal de transparencia de la Municipalidad que son pocas o casi nada las actividades que registran. En el Centro Poblado Pampa Grande cuentan con un nuevo ambiente recreativo en beneficio de la población del CIAM con talleres de capacitación permanente de manualidades y repostería complementaria y la única actividad física sería el Tai Chi, todo es totalmente gratuito, sin embargo esta localidad pertenece a la Zona I - Pachacamac Histórico.

Según fuentes del INEI. Pachacamac geográficamente el $90 \%$ está compuesto netamente por Centros Poblados Rurales (CPR) Divididos en V zonas. La zona III. Se le conoce como CPR "Centros Poblados Rurales" cuenta con 11 (CPR); y la zona IV llamada "José Galvez Barrenechea" cuenta con 1(CPR); la zona V "Huertos de Manchay" cuenta con 22 (CPR). Cada CPR, tienen un aproximado de 127 varones y 97 mujeres entre 45 a 65 años de edad, teniendo un promedio para el desarrollo del estudio en razón de los CPR.

Según la OMS dice que la vejez es un proceso inevitable el cual sigue su ciclo natural en cada ser humano, incide en diferentes aspectos de la vida, siendo en la mayoría de estos desfavorable en la medida que el ser humano pierde autonomía, atentando contra su integridad física, mental y social. Algunas de las características que se presenta en esta etapa de la vida son el sedentarismo y la inactividad física, los cuales traen como consecuencia la presencia de alteraciones fisiológicas y patológicas.

En el distrito de Pachacamac, dentro los (CPR), se descubrió que su población de la tercera edad, tiene una serie de deficiencias fisiológicas y patológicas debido a su inactividad física y estilo de vida sedentario, y dado que es un grupo vul- nerable en extrema pobreza, se propuso aplicar el estudio, teniendo como objetivo; Desarrollar ejercicios aeróbicos acuáticos para reducir el deterioro del sistema muscular, mejorar la calidad de vida de las personas mayores en las zonas rurales (CPR) del distrito de Pachacamac.

La actividad física, aplicada correctamente y con un programa adecuado, beneficia a una persona mayor que trabaja en etapas, ya que el envejecimiento, como se indica, es natural. Chirinos (2017) introdujo un programa de actividad física para reducir el riesgo de caídas en los ancianos en 2015 - 2016, para lograr resultados efectivos y positivos para la población.

Otra razón para los ancianos es la pérdida de fuerza y equilibrio, ya que son irreversibles para la recuperación, por lo que tenemos talleres de agua que mejoran su estabilidad y, por lo tanto, mantienen una fuerza moderada para su edad y condición física. Como parte de la investigación; Chalapud L. y Escobar A. (2017).

Presentaron el programa "Actividad física para mejorar la fuerza y el equilibrio en las personas mayores". Donde revelan que la tercera edad cubre un período bastante amplio de la vida de una persona, y la actividad física es una estrategia que puede mejorar la calidad de vida de este grupo de la población. El propósito del estudio fue determinar la efectividad de un programa de actividad física para mejorar la fuerza y el equilibrio de las extremidades inferiores en el adulto mayor.

Se ha demostrado con éxito que a través del programa, la fuerza y el equilibrio mejora en las personas mayores. Esto muestra que el trabajo ordenado y bien programado mejora la calidad de vida de las personas; y aceptar estos estudios y la capacidad de aplicar obras de agua (piscina); el estudio fue tomado de; Ruiz y Goyes (2015).

En aquellos casos en que realizan estudios sobre actividad física recreativa en personas mayores, en los que demuestran que aumentar la esperanza de vida es uno de los problemas urgentes del 
mundo, comenzando, en primer lugar, por impartir calidad de vida y deportes recreativos y uso del agua. tipos de deportes por etapas, con resultados positivos, ya que el estudio considera elementos fundamentales como la evaluación de la composición corporal (altura, peso, circunferencia de la cintura y pliegues), la condición física (flexibilidad, equilibrio estático, resistencia y fuerza, de acuerdo con la batería del Senior Fitness Test) y la calidad la vida de las personas mayores (pestaña "WHOQOL-100"); luego aplique el programa de acuerdo con el diagnóstico; Sus resultados fueron óptimos, se redujo el peso corporal, mejoró la condición física; Las vacaciones con sentido educativo han mejorado su calidad de vida.

Teniendo en cuenta los antecedentes y cómo lograron mejorar la calidad de vida y los problemas de salud física en las personas mayores. El estudio tiene esta similitud, dado que la población tiene otras características, como la desnutrición, un estilo de vida sedentaria y la pobreza extrema, y según la observación, se propuso desarrollar un programa acuático en Piscina, con cuatro actividades consecutivas, la primera acción es el proceso de socialización, rehabilitación en piscinas y trabajo con drilles, así como control del cuerpo;

La segunda acción será fortalecer la masa muscular en el agua a través de ejercicios diseñados o terapia motora; El tercer ejercicio es que desarrolle ejercicios aeróbicos de baja intensidad más frecuentes para crear confianza y autoestima a través de actividades recreativas y ejercicios dinámicos, y finalmente, el cuarto ejercicio es tener un trabajo diferenciado en el agua, donde las personas mayores ya están familiarizadas a la natación, según las características del adulto mayor.

Por esta razón, la propuesta del objetivo es mejorar la condición fisiológica y patológica de las personas de mayores en los Centro Poblados Rurales (CPR) del distrito de Pachacamac.

\section{MÉTODOS Y MATERIALES}

El método de investigación acción, tiene un propósito aplicativo y se ha utilizado el enfoque cuantitativo para obtener datos necesarios, que existe en la población de estudio, donde se identifica a las personas de la tercera edad que viven en los CPR (Centro Poblado Rural) "Manchay José Gálvez", del Distrito de Pachacamac; teniendo como objetivo general aplicar el desarrollo de una serie de actividades físicas acuáticas sub aeróbicas de bajo impacto para disminuir el deterioro del sistema muscular y mejorar la calidad de vida de la población de la tercera edad en los CPR, distrito de Pachacamac.

Objetivo específico es diagnosticar el estado médico, nutricional, psicológico y físico del adulto mayor; teniendo como primera actividad el diagnóstico del médico mediante una evaluación médica del sistema muscular; como segunda actividad es la participación del Nutricionista, para desarrollar la evaluación nutricional; la tercera actividad es la participación del Psicólogo para aplicar el test de EVEA y ver su estado emocional, y finalmente el investigador con los resultados obtenidos de cada profesional aplica una evaluación de diagnóstico en las actividades físicas, para tener la ficha general de cada adulto mayor.

El segundo objetivo, que es desarrollar el programa innovador "aqua health" según sus secuencia de las 4 actividades programadas. Proceso de socialización; Proceso de fortalecimiento de masa muscular, Desarrollo de movimiento su aeróbicos y por último trabajo de estilos en la natación para mejorar la calidad de vida en el adulto mayor. Y el último objetivo específico es, Evaluar los efectos de las actividades físicas sobre el estado médico, nutricional, psicológico y finalmente el físico en personas de la terca edad del CPR en el distirto de Pachacamac.

Y finalmente aplicado el estudio tendrá resultado final donde demostrar que el Programa "Aqua Health" mediante las diferentes actividades físi- 
cas aeróbicas de baja intensidad, reducen, disminuyen y previenen el deterioro del sistema muscular y por ende hay mejora de la calidad de vida.

Se aplicó un diseño de investigación cuasi experimental, con un pret test y un post test, porque su propósito es evaluar y examinar y mejorar la calidad de vida del adulto mayor, considerando sus efectos encontrados en la aplicación, en el adulto mayor de los (CPR) cuando realizan una actividad física aeróbicas, es decir, se observarán los fenómenos en su contexto natural, se aplicó la práctica regular de la actividad física por un período 8 meses; durante el tiempo se analizó y demostró la mejora del trabajo propuesto en la actividad física aeróbica y los modos y comportamiento de hábitos del adulto mayor siendo de suma importancia final.

Dentro la técnicas e Instrumentos de Recolección de Datos, se utilizó en primer lugar una ficha de categorías para medir el pre test, de acuerdo a la observaciones obtenidas y se ordenó de acuerdo a rangos de respuestas iniciales, teniendo como intervalos de medición 1) nulo, 2) Escaso, 3) Bueno, 4) Optimo. Si desarrollan actividades físicas para mejorar la calidad de vida en las personas de la tercera edad. Y posteriormente se aplicó la misma ficha para el post test, y ver los cambios encontrados en la muestra de estudio representativa para el proyecto "Aqua Health", demostrando los resultados de la aplicación del programa.

Dentro las evaluaciones secundarias se tuvo, la evaluación médica e historia clínica, el nutricional aplica su test MNA, el psicólogo aplica su Test de EVEA, y finalmente el Investigador aplica el Test de COOPER. Complementado las pruebas para la aplicación del programa. Con los resultados obtenidos se llevó a cabo el programa de "Aqua Health". Aplicado por niveles y epatas, según los diagnósticos. Siendo el primer programa (Aquamedical) o la rehabilitación en agua, por 16 sesiones por 2 meses (dos veces a la semana).

Seguido se aplicó el segundo programa "aquanutricional" o adulto feliz; por 16 sesiones por 2 meses (dos veces a la semana). Seguido se aplicó el tercer programa "aquapsicological" o soy adulto; por 16 sesiones por 2 meses (dos veces a la semana). Y finalmente se aplicó el programa "aquafisical" o vida saludable; por 16 sesiones por 2 meses (dos veces a la semana). Teniendo un trabajo de 8 meses. Finalizado el proyecto se volvió a evaluar con los primero instrumentos, para finalizar el trabajo de campo.

Para la recolección de datos será por medio de fichas, grabaciones, fotografías y los test aplicados todo es evidenciado por cada sesión desarrollada. Las actividades de campo se contó con el apoyo de tesistas y docentes de la escuela Profesional Ciencias del Deporte de la Universidad Alas Peruanas.

La población de estudio fueron 127 varones y 97 mujeres con edades de 45 a 70 años, teniendo población de 224 adultos mayores, la muestra fue representada con margen de error del 5\%, y su nivel de significancia del 95\%. Obteniendo una muestra de 53 adultos mayores. 30 varones y 23 mujeres. Los cuales se sometieron al programa "Aqua Health".

Finalmente se procesó la información y se tabulo para comprar los resultados iniciales con los finales en el estudio, mediante tablas estadísticas. Finalmente entregar el trabajo a los responsables sociales sobre los resultados logrados de los Centros Poblados Rurales (CPR) distrito de Pachacamac, el cual serán datos importantes para su base de datos en el CIAM del Distrito de Pachacamac respectivamente.

\section{RESULTADOS}

Los resultados obtenidos del programa "Aqua Health". Se demostraron de la siguiente. Teniendo una Hipótesis general, que dice sí; Las actividades físicas acuáticas sub aeróbicas de bajo impacto disminuyen el deterioro del sistema muscular y mejoran la calidad de vida de la población de la tercera edad en los CPR, distrito de Pachacamac. 


\section{Análisis descriptivo}

Figura $\mathrm{N}^{\circ}$ 01: Desarrollo de las actividades físicas acuáticas sub aeróbicas de bajo impacto en el Pre test

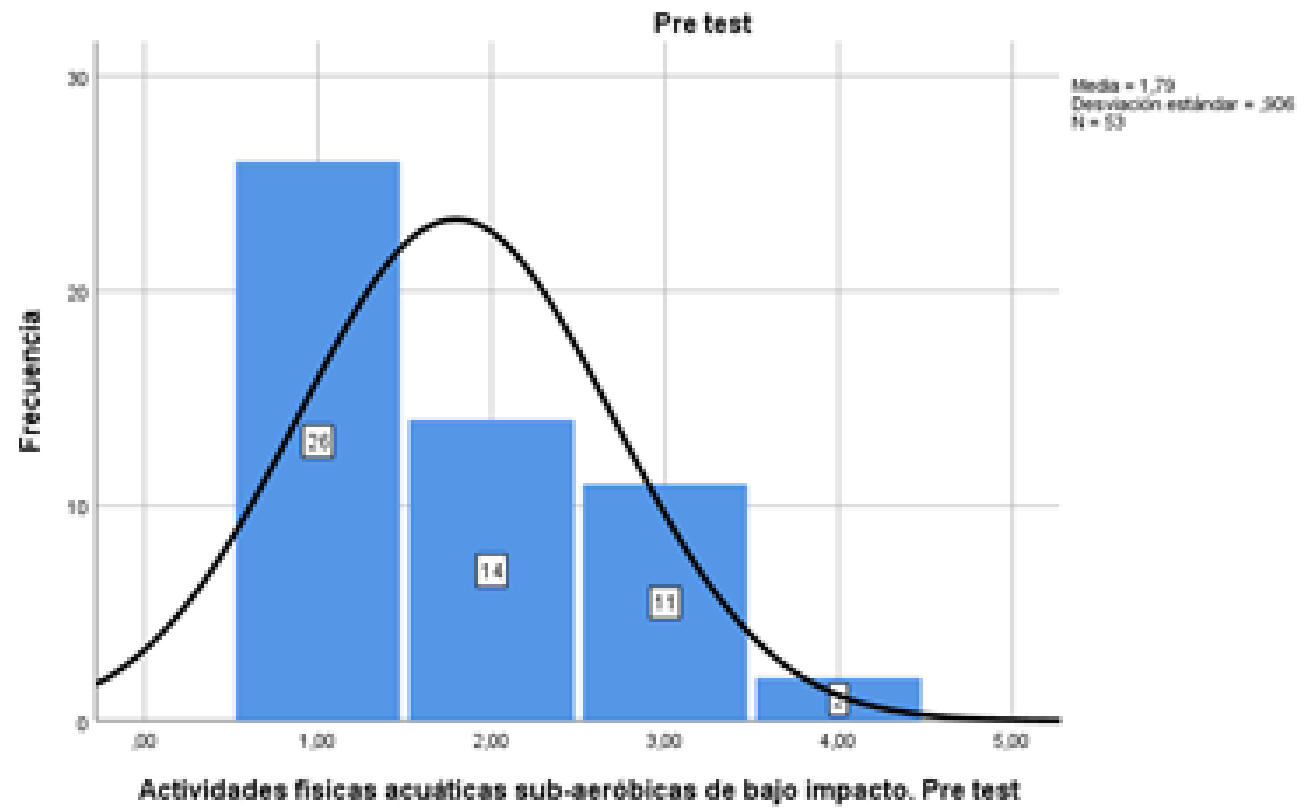

Interpretación: En la figura $\mathrm{N}^{\circ}$.1. Se observa que, el 26 adultos mayores su actividad física es nula; 14, adultos escasa; 11 adultos mayores es buena, y 2 Adultos mayores es óptima en el desarrollo de las actividades físicas acuáticas sub aeróbicas de bajo impacto, aplicados en el Pre test.

Figura $\mathrm{N}^{\circ}$ 02: Desarrollo de las actividades físicas acuáticas sub aeróbicas de bajo impacto en el Post test

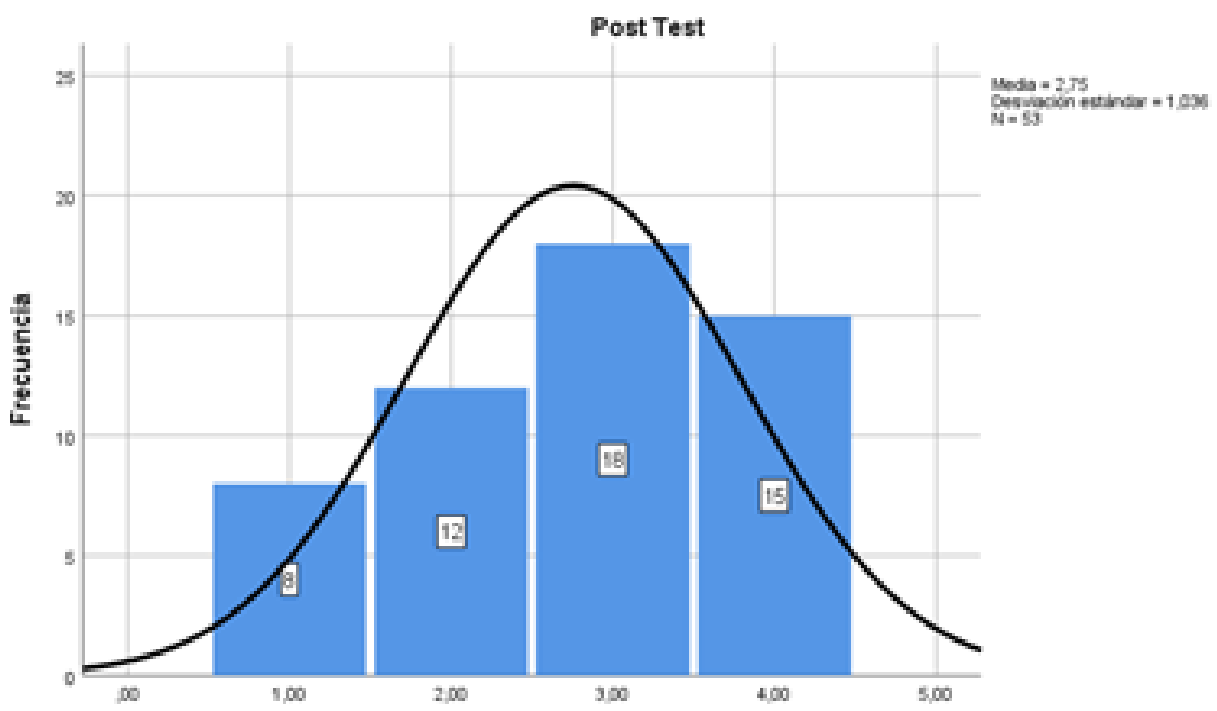

Actividades fisicas acuaticas sub-aerobicas de bajo impacto. Pos test 
Interpretación: En la figura $\mathrm{N}^{\circ} .2$. Se observa que, 8 adultos mayores su actividad física es nula; 12 , adultos escasa; 18 adultos mayores es buena, y 15 Adultos mayores es óptima en el desarrollo de las actividades físicas acuáticas sub aeróbicas de bajo impacto, aplicados en el Post test.

\section{Análisis Inferencial}

H1: Las actividades físicas acuáticas sub aeróbicas de bajo impacto disminuyen el deterioro del sistema muscular y mejoran la calidad de vida de la población de la tercera edad en los CPR, distrito de Pachacamac.

H0: Las actividades físicas acuáticas sub aeróbicas de bajo impacto no disminuyen el deterioro del sistema muscular y mejoran la calidad de vida de la población de la tercera edad en los CPR, distrito de Pachacamac.

\begin{tabular}{|c|c|c|c|c|c|}
\hline \multicolumn{6}{|c|}{ Estadisticas de muestras relacionadas } \\
\hline & & Media & $\mathrm{N}$ & Desv. Desviación & Desv. Error promedio \\
\hline Pre test & $\begin{array}{l}\text { Actividades fisicas acuáticas sub- } \\
\text { aeróbicas de bajo impacto. }\end{array}$ & 1,7925 & 53 &, 90636 &, 12450 \\
\hline Post test & $\begin{array}{l}\text { Actividades fisicas acuáticas sub- } \\
\text { aeróbicas de bajo impacto. }\end{array}$ & 2,7547 & 53 & 1,03600 &, 14231 \\
\hline
\end{tabular}

\begin{tabular}{|c|c|c|c|c|}
\hline \multicolumn{5}{|c|}{ Correlaciones de muestras relacionadas } \\
\hline & & $\mathrm{N}$ & Correlación & Sig. \\
\hline $\begin{array}{l}\text { Pre test } \\
\text { Post test }\end{array}$ & $\begin{array}{l}\text { Actividades fisicas acuáticas sub- } \\
\text { aeróbicas de bajo impacto. } \\
\text { Actividades fisicas acuáticas sub- } \\
\text { aeróbicas de bajo impacto. }\end{array}$ & 53 & 825 &, 000 \\
\hline
\end{tabular}

\begin{tabular}{ll|c|c|c|c|c|c|c}
\hline \multicolumn{7}{c}{ Prueba de muestras relacionadas } \\
\hline
\end{tabular}

Interpretación de resultados: Los participantes al inicio de la aplicación del programa experimentaban actividades físicas acuáticas sub aeróbicas de bajo impacto, significativamente menores antes del tratamiento del programa $(\mathrm{M}=1.7925, \mathrm{SE}=0.12)$ que después del mismo $(\mathrm{M}=2.7547, \mathrm{SE}=0.14, \mathrm{t}$ $(52)=-11.93,<-.05, r=.90)$. Quedando demostrado que las actividades físicas acuáticas sub aeróbicas de bajo impacto después de su aplicación mejoraron significativamente el deterioro del sistema muscular y la calidad de vida de la población de la tercera edad en los CPR, distrito de Pachacamac. 


\section{DISCUSIÓN}

El estudio permite incluir un programa diferenciado en el sector de la población vulnerable con resultados óptimos. Según; Chirinos (2017) presenta un programa de actividad física para reducir el riesgo de caídas en los ancianos en 2015 - 2016, con resultados positivos. Y de la misma manera; Chalapud L. y Escobar A. (2017) están desarrollando otro programa de actividad física para mejorar la fuerza y el equilibrio en las personas mayores, lo que conduce a una mejora en la calidad de vida de su población.

Y finalmente; Ruiz y Goyes (2015) realizan una investigación sobre la actividad física recreativa en las personas mayores para aumentar la esperanza de vida en las personas mayores, logrando resultados positivos en su programa destinado a evaluar la composición corporal y la condición física. Los resultados obtenidos fueron óptimos $y$, por lo tanto, mejoraron la calidad de vida en su población.

El estudio presenta un programa de ejercicio subaeróbico en agua de baja intensidad para mejorar el deterioro del sistema muscular y la calidad de vida de los ancianos en la Centros Poblados Rurales (CPR), Distrito de Pachacamac, con resultados significativos. Uno de los factores de evaluación fue el trabajo con una población mixta de ancianos y en condiciones de extrema pobreza en su totalidad, donde el programa se enfoca más en la actividad en agua (piscina), que fue el resultado de la simpatía y el conocimiento positivo.

La evidencia es notoria, dado que existen programas de beneficios, es positiva, el estudio ha demostrado ser muy útil. Por lo tanto, los datos pueden verificarse para la reproducción social entre los ciudadanos en las mismas condiciones.

\section{CONCLUSIONES}

Se ha desarrollado un programa de actividades físicas sub-aeróbicas de bajo impacto para dismi- nuir el deterioro del sistema muscular y mejorar la calidad de vida en personas de la tercera edad, en los Centros Poblados Rurales. Distrito de Pachacamac.

Se concluye que el programa fue de gran ayuda en la población porque se incrementó la valoración social y humana en las personas de la tercera en los CPR Pachacamac. Considerado algo negativo para muchas personas.

Se concluyó que desarrolla un diagnostico antes y después del estado médico, nutricional, psicológico y físico en personas de la tercera edad, contribuyen, a obtener datos favorables los cuales acompañan al programa con regímenes de control y posición humana en las personas de la tercera edad. Y de la misma manera concientizar a la población que la vida se valora en cualquier epata de condición.

Se concluye que el programa "Aqua Health" cuenta con 4 etapas evolutivas (Aquamedical, Aquanutricional, Aquapsicológical y Aquafisical) logrando resultados favorables en la Población del adulto mayor

Se concluye que el programa "Aqua Health" puede ser aplicado a otras poblaciones similares y en el distrito como en otros lugares del Perú que, cuenta con las mismas características médicas, psicológicas, nutricional y física, con mucha necesidad de ser atendidas.

\section{REFERENCIAS BIBLIOGRÁFICAS}

1. Bermejo, L. (2010). Envejecimiento Activo y Actividades Socioeducativas con personas Mayores. Madrid - España: Editorial Médica Panamericana. Recuperado de: https:// www.siis.net/documentos/Digitalizados/Envejecimiento $\% 20$ Activo\%20y\%20Actividades\%20Socioeducativas. pdf

2. Chalapud-Narváez LM, Escobar-Almario A. (2017). Actividad física para mejorar fuerza y equilibrio en el adulto mayor. Universidad y Salud, Sección de Artículos Originales, 19(1):94-101. Recuperado desde: http://dx.doi. org/10.22267/rus.171901.73

3. Chirinos J. (2017). Programa de actividad física para disminuir el riesgo de caídas del club adulto mayor Men- 
tes Activas del Centro de Salud Ganimedes 2015 - 2016. Universidad Cesar Vallejo, Lima, Perú. Recuperado: http:// repositorio.ucv.edu.pe/bitstream/handle/UCV/9111/Chirinos_AJP.pdf? sequence $=1$ \&isAllowed $=\mathrm{y}$

4. Escobar, J. (2003). Intervención Pedagógica y Didáctica en Adultos y Adultos Mayores para la Actividad Física. Buenos Aires - Argentina: Revista Digital EF Deportes. Recuperado de: https://www.efdeportes.com/efd63/adultos.htm

5. González, J.M. (2000). Indicaciones y Sugerencias sobre el Entrenamiento de Fuerza y Resistencia en Ancianos. Córdoba - Argentina: Revista Internacional de Medicina y Ciencias de la Actividad Física y el Deporte. Recuperado de: http://www.ilustrados.com/tema/7000/Importancia-Actividad-Fisica-Tercera-Edad.html

6. Hernández, R. y Col. (2014). “Metodología del a investigación”. México: Mc Graw Hill. Recuperado de https:// www.uv.mx/personal/cbustamante/files/2011/06/Metodologia-de-la-Investigaci\%C3\%83\%C2\%B3n_Sampieri.pdf

7. Instituto Nacional de Estadística e Informática del Perú. https://www.inei.gob.pe/

8. Ley No 30490 de la persona adulta mayor.

9. Lloret, M., C. Conde, J. Fagoaga, C. León, C. Tricas. (2004). Natación Terapéutica. Barcelona - España: Editorial Paidotribo. Recuperado de: https://www.ugr. es/ swimsci/SwimmingScience/page4/page16/page59/files/IIcongreso\%20mundial\%20CCAFD.pdf

10. Marcos Pardo. P; Orquín Castrillón, J; Belando Pedreño, N. y Moreno-Murcia, J. (2014). Motivación auto determinada en adultos mayores practicantes de ejercicio físico. Universidad de Murcia. España. Recuperado de. http:// scielo.isciii.es/pdf/cpd/v14n3/art015.pdf

11. Municipalidad Distrital de Pachacamac. Centro Integrales de Atención a la Persona Adulta Mayor (CIAM). http://www.munipachacamac.gob.pe/ciam.html\#

12. Ordenanza Municipal 169-2016-MDP/C proyecto de Ordenanza que aprueba la Creación del Centro Integral del Adulto Mayor -CIAM

13. Organismo Mundial de Salud. https://www.who.int/es 14. Ortega, Ricardo, Sánchez, Pinilla. (1992). Medicina del Ejercicio Físico y del Deporte para la Atención a la Salud; Madrid - España: Editorial Díaz de Santos, S.A

15. Ruíz O., L. A. \& Goyes E., L. A. (2015). Actividad física recreativa en el adulto mayor. Educación Física y Deporte, 34 (1), 239-267. Ene-Jun. Recuperado: http://doi. org/10.17533/udea.efyd.v34n1a11 